\title{
Sollen sich die Hilfswerke aus der operativen Entwicklungszusammenarbeit zurückziehen?
}

\section{Peter Niggli}

\section{(2) OpenEdition}

12 Journals

Electronic version

URL: http://journals.openedition.org/sjep/507

DOI: 10.4000/sjep.507

ISSN: 1663-9677

Publisher

Institut de hautes études internationales et du développement

Printed version

Date of publication: 1 novembre 2004

Number of pages: 141-148

ISBN: 2-88247-057-6

ISSN: $1660-5926$

\section{Electronic reference}

Peter Niggli, « Sollen sich die Hilfswerke aus der operativen Entwicklungszusammenarbeit zurückziehen? », Schweizerisches Jahrbuch für Entwicklungspolitik [Online], 23-2 | 2004, Online erschienen am: 10 Juni 2010, abgerufen am 08 September 2020. URL : http:// journals.openedition.org/sjep/507 ; DOI : https://doi.org/10.4000/sjep.507 


\section{Sollen sich die Hilfswerke aus der operativen Entwicklungszusammenarbeit zurückziehen?}

\section{Peter Niggli*}

| $\mathrm{n}$ verschiedenen Industrieländern stehen die Beziehungen zwischen privaten Entwicklungsorganisationen und staatlichen Entwicklungsagenturen unter Druck. Selbstverständliche Formen der Zusammenarbeit und der staatlichen Mitfinanzierung von Hilfswerken werden hinterfragt. Hauptsächlicher Grund sind die Veränderungen in der Praxis und der Konzeption der Entwicklungszusammenarbeit (EZA). Eine wichtige Rolle spielen dabei (1) Gewichtsverschiebungen zwischen nördlichen Hilfswerken und Nichtregierungsorganisationen (NRO) der Entwicklungsländer, (2) die stärkere Gewichtung des Staates der Entwicklungsländer durch die Geberländer sowie (3) die Absicht international tätiger Unternehmen, an die Entwicklungshilfegelder der reichen Länder zu kommen.

\section{Veränderungen in der Entwicklungszusammenarbeit}

(1) Es ist heute gängige Praxis, dass sowohl die staatlichen Entwicklungsagenturen, als auch die privaten Hilfswerke mit Süd-NRO zusammenarbeiten und diese (mit)finanzieren. Für die Hilfswerke ist dies seit längerem Tradition; für die zuständigen Regierungsstellen ist es eine Praxis, die im Laufe der neunziger Jahre an Gewicht gewonnen hatte. Einzelne staatliche Entwicklungsbürokratien fragen deshalb, wieso es überhaupt noch private Hilfswerke im Norden brauche. Es gebe in den Entwicklungsländern heute viele qualifizierte $\mathrm{NRO}$, mit denen die Geberstaaten direkt zusammenarbeiten könnten. Es sei nicht mehr nötig, solche Süd-NRO über nördliche Hilfswerke zu unterstützen. Dies verursache nur höhere Transaktionskosten. Diese Ansicht ist auch in den Koordinationsbüros der Direktion für Entwicklung und Zusammenarbeit (DEZA) verbreitet.

(2) In der zweiten Hälfte der achtziger Jahre wurden die staatlichen Entwicklungsagenturen wegen ihrer Zusammenarbeit mit den Regierungen der Entwicklungsländer heftig kritisiert. Die Hilfe fliesse in grössenwahnsinnige staatliche Entwicklungsvorhaben, die am Los der benachteiligten Schichten in diesen Ländern nichts änderten, es manchmal sogar verschlechterten und wenig bis nichts zum Wachstum der Wirtschaft beitrügen. Die Kritik verstärkte sich mit dem Ende des Kalten Kriegs, da allzu grosse Teile der staatlichen Entwicklungshilfe nur dazu gedient hätten, befreundete Regierungen an der Macht zu halten. Vielerorts wurde daraus die Lehre gezogen, öffentliche Entwicklungsgelder zunehmend über nördliche Hilfswerke fliessen zu lassen und in den Entwicklungsländern vermehrt NRO, und weniger die

* Geschäftsleiter der Arbeitsgemeinschaft Swissaid/Fastenopfer/Brot für alle/Helvetas/Caritas/HEKS und Leiter des Bereichs Entwicklungspolitik. 
Regierungen zu unterstützen. Die antistaatliche Wende der Entwicklungszusammenarbeit passte zur ersten Generation marktradikaler Strukturanpassungsmassnahmen der Bretton-Woods-Institutionen. Den nördlichen Hilfswerken fiel in diesem Modell die Aufgabe zu, die Entstehung von NRO im Süden zu fördern (,Förderung einer lebendigen Zivilgesellschaft“) und deren Handlungsfähigkeit zu stärken (Capacity building). Heute sind die Akzente verschoben. Ein schwacher Staat gilt - zu Recht - als Entwicklungshindernis. Und südliche NRO werden nicht mehr als tauglicher Ersatz für einen funktionsunfähigen Staat betrachtet. Die Regierungen der OECD-Staaten favorisieren jetzt, zumindest in der Theorie, eine Konzentration der Hilfe auf ausgewählte Entwicklungsländer, deren Regierungen sich im Rahmen der Standards des Entwicklungshilfeausschusses der OECD (DAC) wohlverhalten. Die Geberländer wollen daher wieder mehr Mittel direkt über das Staatsbudget und über staatlich koordinierte Programme der Entwicklungsländer fliessen lassen und lokale Behörden stärken. Diese neue Bündelung der Hilfsgelder geht tendenziell auf Kosten der nördlichen Hilfswerke und etwas weniger - der südlichen NRO, sofern diese keine Funktion in den Entwicklungsplänen der jeweiligen Regierung und der Konferenz der Geberländer finden. Auch die DEZA und die Entwicklungsabteilung des Staatssekretariats für Wirtschaft (seco) wollen sich vermehrt auf Budgethilfe und koordinierte Einflussnahme der Geberländer auf das jeweilige Staatsbudget konzentrieren. $\mathrm{Zu}$ einem grossen Teil wird dieser Prozess in den ärmsten Ländern durch die Armutsreduktionsstrategien vorangetrieben, welche die Länder erarbeiten müssen, um weiterhin Kredite von Weltbank und Internationalem Währungsfonds erhalten zu können.

(3) Gleichzeitig drängen neue Konkurrenten aus der Privatwirtschaft an die Töpfe der Entwicklungshilfebudgets der Geberländer. Im Zeichen der Millenniums-Entwicklungsziele propagieren die Geberländer - durch die Weltbank sekundiert -, wieder vermehrt in die Infrastrukturen der Entwicklungsländer zu investieren. Das war schon im Kalten Krieg ein Schwerpunkt der Entwicklungshilfe gewesen, der dann in der allgemeinen Kritik an der staatlichen Entwicklungshilfe an Gewicht verloren hatte. Heute propagieren die OECD-Länder für den Infrastrukturausbau die „Partnerschaft“ mit geeigneten Privatunternehmen, um den Staat der Entwicklungsländer „schlank“ zu halten. Theoretisch soll damit zusätzliches privates Kapital für die Entwicklung der armen Länder mobilisiert werden. In der Praxis werden die Industrieländer (und die „begünstigten“ Entwicklungsländer) Investitionsbeihilfen aller Art zur Verfügung stellen müssen, um den privaten Partnern die Angst vor dem Risiko zu nehmen. Diese staatliche Investitionsförderung droht zulasten anderer Posten der Entwicklungshilfebudgets zu gehen, also auch zulasten der Hilfswerke und der Süd-NRO.

$\mathrm{Zu}$ diesen sozusagen sachlichen Gründen für den Wandel der Beziehungen zwischen nördlichen Hilfswerken und staatlichen Entwicklungsagenturen kommen politische Verstimmungen hinzu. Manche Regierungen stören sich an der anwaltschaftlichen Öffentlichkeitsarbeit und Interessenvertretung (Advocacy) sowie an der wirtschaftspolitischen Kritik der Hilfswerke, vor allem seit sie parallel zur internationalen Protestbewegung gegen die wirtschaftliche Globalisierung läuft. In der Schweiz war etwa der Bundesrat empört darüber, dass sich die Hilfswerke nicht deutlich von der Gewalt an den Demonstrationen gegen 
das Weltwirtschaftsforum (WEF) distanzierten. Diese fühlten sich dazu nicht veranlasst, weil sie nicht an den Massenkundgebungen beteiligt waren und auch nicht dazu aufgerufen hatten. Da aber die Arbeitsgemeinschaft der Hilfswerke die Politik der permanenten Liberalisierung immer wieder als entwicklungsschädlich kritisiert hatte, waren die Hilfswerke in den Augen des Bundesrats gewissermassen automatisch mit dem Protest gegen das WEF verknüpft.

In einzelnen Ländern sorgten die bewaffneten Interventionen in Afghanistan und im Irak für Zündstoff. Die Bush-Regierung empfindet es als drastischen Affront, dass amerikanische Hilfswerke dem Feldzug gegen den Terrorismus skeptisch gegenüberstehen und die systematische Vermischung von militärischen und humanitären Aktionen scharf kritisieren. In Grossbritannien und bei anderen Mitgliedern der „Koalition der Willigen“ beteiligten sich die Hilfswerke am Protest gegen die Teilnahme am Irakkrieg. Sie standen damit im Einklang mit der Mehrheit der Bevölkerung ihrer Länder, handelten sich aber Ärger mit den Regierungen ein ${ }^{1}$.

Ungeachtet solcher politischer Irritationen sehen sich die staatlichen Entwicklungsagenturen gezwungen, die anwaltschaftliche Öffentlichkeitsarbeit der nördlichen Hilfswerke grundsätzlich als berechtigte Tätigkeit anzuerkennen. Die Geberländer können schlecht von den Entwicklungsländern mehr politischen Spielraum für die Zivilgesellschaft verlangen, um diesen dann im Gegenzug den Hilfswerken zu verwehren. Ganz abgesehen davon, dass einer versuchten Bevormundung der Nord-NRO die verfassungsmässig garantierten Grundrechte in den Geberstaaten entgegenstünden. Einige Entwicklungsagenturen verlangen sogar, dass die Hilfswerke mehr in die Advocacy investieren. Dieser Ruf ist auch innerhalb der DEZA vernehmbar. Es gibt dort Stimmen, welche meinen, die Hilfswerke sollen sich ganz auf Advocacy konzentrieren und das operative Geschäft der Entwicklungszusammenarbeit dem Staat überlassen.

Für die DEZA waren unter anderem solche Diskussionen Anlass, ihre NROPolitik im Jahr 2004 extern evaluieren zu lassen. Bei Redaktionsschluss stand ein Evaluationsbericht zur Diskussion, welcher tiefgreifende Änderungen im Verhältnis der DEZA zu den Hilfswerken vorschlägt und viel Zündstoff enthält. Vor diesem Hintergrund möchte ich einige Punkte skizzieren, die meines Erachtens für das Verhältnis von Hilfswerken und staatlichen Entwicklungsagenturen in der Schweiz und darüber hinaus wichtig sind.

\section{Welche Arbeitsteilung zwischen den Hilfswerken und der DEZA?}

1. In vielen Industrieländern ist es Tradition, dass öffentliche Aufgaben durch staatliche und gemeinnützige private Träger gemeinsam wahrgenommen werden. Letztere mobilisieren dafür oft eigene Spendengelder, werden aber zusätzlich durch die öffentliche Hand mitfinanziert. Die Schweiz ist diesbezüglich keine Ausnahme. Im Gegenteil, das Netz privater Organisationen im Dienste öffentlicher Aufgaben ist hierzulande besonders dicht. Natürlich stellt sich dabei immer wieder die Frage, wie die Aufgaben zwischen öffentlichen und privaten Trägern verteilt werden sollen. Das gilt auch für die Hilfswerke und die DEZA.

1 Die politischen Spannungen rund um die globalisierungskritische Bewegung mündeten in eine internationale Diskussion über die Legitimation von NRO, das Handeln demokratisch gewählter Regierungen zu hinterfragen. Siehe den Artikel : „Haben die NRO ein Legitimitätsproblem?“ in diesem Dossier. 
Nicht zur Diskussion steht aber meines Erachtens der Grundsatz, dass die öffentliche Aufgabe Entwicklungszusammenarbeit auch durch die privaten Hilfswerke wahrgenommen werden soll. Die Hilfswerke bringen dazu namhafte eigene Geldmittel ein und arbeiten in der Regel billiger als die entsprechenden staatlichen Instanzen. Sie verfügen über ein vergleichbares Know-how und über eigene zivilgesellschaftliche Kontakt- und Partnernetze in den jeweiligen Ländern. Es gibt weder einen politischen noch einen finanziellen Grund, die öffentlichen Entwicklungsgelder nur durch die staatlichen Entwicklungsagenturen verwalten zu lassen.

Einige Personen in der DEZA halten die Hilfswerke für überflüssig. Sie stellen sich vor, dass die DEZA die Funktionen der Hilfswerke in der bilateralen Entwicklungszusammenarbeit übernehmen kann. Man kann diese Diskussion schon führen, muss dann aber auch das Umgekehrte erwägen: Funktionen in der bilateralen EZA, die heute die DEZA erfüllt, könnten auch die Hilfswerke übernehmen. Im Prinzip können alle Aufgaben der Entwicklungszusammenarbeit, die nicht zwingend die Intervention der staatlichen Agentur verlangen, an Hilfswerke und NRO übertragen werden. Das wäre einiges mehr als das, was die Hilfswerke bis jetzt tun. Ein solcher Entscheid könnte als Schlankheitskur für die grosse Bürokratie, welche die DEZA unzweifelhaft darstellt, verkauft werden. Er entspräche dem gegenwärtigen Zeitgeist des Rundumsparens und Staatsabbaus, und ist nur deswegen nicht aktuell, weil den politischen Kräften, welche einen solchen Entscheid begrüssen könnten, die Hilfswerke nicht besonders nahe stehen.

2. Die Entwicklungszusammenarbeit ist nur eine von vielen öffentlichen Aufgaben. Im Unterschied zu anderen öffentlichen Aufgaben ruht ihre innenpolitische Legitimation auf zarten Fundamenten, da die Bürgerinnen und Bürger keinen erkennbaren Vorteil daraus ziehen. Die EZA geht auch keine sichtbaren oder erfahrbaren Probleme an, die alle betreffen. Entwicklungszusammenarbeit beruht damit innenpolitisch gänzlich auf ideellen und ethischen Motiven der Bevölkerung. In der Schweiz ist dieses ideelle Fundament vergleichsweise breit. Es gab und gibt eine breite Strömung in der Bevölkerung - nicht zuletzt gestützt durch die zwei Landeskirchen -, der die Hilfe zur Selbsthilfe für die Benachteiligten dieser Welt am Herzen liegt ${ }^{2}$. Allerdings garantiert diese Strömung nicht in jedem Fall politische Mehrheiten ${ }^{3}$. Das ist allen politischen Kräften bewusst, auch denjenigen, die über einen grösseren Kahlschlag im EZABudget nachdenken.

Die Hilfswerke repräsentieren in einem gewissen Mass das organisatorische Rückgrat der EZA-freundlichen Strömung und beleben sie. Die DEZA kann diese Aufgabe nicht übernehmen und die Hilfswerke in dieser Funktion nicht ersetzen. Aber sie sollte ein grosses Interesse daran haben (und ihr Direktor hat es), dass es weiterhin starke Hilfswerke gibt, und dass diese mit der praktischen

2 Ich anerkenne durchaus, dass Entwicklungszusammenarbeit auch strategisch begründet werden kann und soll. Es ist völlig richtig zu behaupten, dass es im langfristigen nationalen Interesse der Schweiz liegt, die Nord-Süd-Kluft zu verringern und allen Menschen ein Leben in Würde zu ermöglichen.

3 In den regelmässig erhobenen Meinungsumfragen über die Entwicklungszusammenarbeit, welche die DEZA und die Arbeitsgemeinschaft der Hilfswerke durchführen, sprechen sich klare Mehrheiten für die Entwicklungszusammenarbeit aus. Eine ebenso klare Mehrheit spricht sich aber auch dafür aus, einen Teil der Entwicklungsgelder den „Bedürftigen im Inland“ zukommen zu lassen. 
Entwicklungszusammenarbeit verknüpft bleiben. Diejenigen in der DEZA, welche auf die Hilfswerke verzichten wollen, untergraben das innenpolitische Fundament der öffentlichen Aufgabe Entwicklungszusammenarbeit.

3. Dass die Geberländer heute dem Staat in den Entwicklungsländern wieder eine grössere Rolle zutrauen und mehr Hilfe über die Regierungen und deren Entwicklungspläne fliessen lassen - meines Erachtens durchaus zu Recht -, rechtfertigt keine EZA-Strategie, in der alles nur noch auf diese Karte gesetzt wird. Eine Investition auf der Gegenseite, zur Stärkung der „Zivilgesellschaft“, ist weiterhin nötig, und zwar auch aus öffentlichen Mitteln. Dafür gibt es zwei Gründe.

Erstens kann Budgethilfe nur in Ländern geleistet werden, die über einigermassen funktionierende Institutionen verfügen und eine akzeptable Regierungsführung vorweisen können. Es wird aber, angesichts des Versagens von Staaten, immer notwendiger werden, auch in Ländern mit schlechter Regierungsführung oder in gescheiterten Staaten nicht nur Nothilfe, sondern auch so etwas wie Entwicklungszusammenarbeit leisten zu können. Hier ist der Weg über Hilfswerke und lokale NRO der Sinnvollste.

Zweitens ist Entwicklung ein Prozess mit sozialen Auseinandersetzungen. Es ist eine Illusion anzunehmen, dass Staaten mit guter Regierungsführung - durch Budgethilfe, Geberkoordination und „Multistakeholder-Partnerships“ für den Infrastrukturausbau aufgerüstet - der Unterstützung der armen Bevölkerung Priorität einräumen. Nicht nur in der Schweiz, sondern auch in den Entwicklungsländern leihen die Regierungen zuerst den wirtschaftlich und politisch Mächtigsten ihr Ohr. Sofern sich die Ärmsten nicht so laut wie möglich selber melden können, drohen sie - „Geber-Konferenzen“ hin oder her - billig abgespeist oder ganz vergessen zu werden.

Den staatlichen Entwicklungsagenturen ist dies wohl bewusst. Deshalb verlangen sie im schwammigen Jargon der internationalen Zusammenarbeit immer wieder die „Partizipation“ der Bevölkerung. Daraus schliesse ich folgendes: Wenn mit EZA-Geldern die staatliche Seite gestärkt wird, so muss nur schon zum Ausgleich auch die Seite der ärmsten Bevölkerungsschichten und der Zivilgesellschaft im Allgemeinen unterstützt werden. Dazu eignen sich die Hilfswerke und lokale soziale Bewegungen oder Organisationen, welche diese Interessen vertreten, nicht jedoch die Geberkonferenzen, staatlichen Entwicklungsagenturen und Regierungen der Entwicklungsländer. Wenn diese die „Partizipation“ der Zivilgesellschaft als Bestandteil in ihre Landesprogramme aufnehmen, dann sollen sie sich auf die Bereitstellung institutioneller Mechanismen konzentrieren, in denen die „Partizipation“" stattfinden kann. Hingegen ist es nicht ihre Aufgabe, die Meinungsfindung, Organisierung und Interessenvertretung der Zivilgesellschaft zu „unterstützen“ und damit zu steuern. Die „beteiligte Zivilbevölkerung“ muss nämlich in der Lage sein, die makroökonomischen Strategien, welche die Geber in diesen Ländern durchsetzen, zu hinterfragen und Druck zur Veränderung staatlicher Politiken aufzubauen.

4. Die Hilfswerke in der Schweiz und anderswo würden es vorziehen, wenn sich die staatlichen Entwicklungsagenturen auf die Zusammenarbeit mit den Behörden der Entwicklungsländer konzentrierten, und zwar auf allen Stufen bis hinunter zu den Lokalbehörden. Den Hilfswerken würden die Beziehungen zu den Süd-NRO überlassen. Auch die Unterstützung von Süd-NRO aus Mitteln der 
öffentlichen EZA würde in dieser Wunschwelt ausschliesslich über die Hilfswerke des Nordens erfolgen. In der Schweiz galt dies übrigens bis in die achtziger Jahre als Grundsatz.

Seither hat die DEZA, wie andere staatliche Entwicklungsagenturen auch, vielfältige direkte Beziehungen zu Süd-NRO und anderen zivilgesellschaftlichen Akteuren aufgebaut. Oft handelt es sich dabei um Akteure, die soziale Leistungen für die Allgemeinheit anbieten, oder um Institutionen und Unternehmen, welche in der Förderung von Kleinunternehmen, neuen Erwerbsmöglichkeiten und ökologischen Produktionsweisen eine Rolle spielen. Das hat sicherlich positiv zur Qualität der staatlichen Entwicklungszusammenarbeit beigetragen. Trotzdem ist es nötig, im Bereich der Beziehungen zur südlichen Zivilgesellschaft wieder zu einer klaren Rollenteilung zwischen DEZA und Hilfswerken zu kommen.

Falls die DEZA heute in einzelnen Ländern vermehrt über Geberkoordination und Budgethilfe in die staatlichen Entwicklungspläne investieren will (oder es schon tut), muss sie sich auch wieder mehr der Arbeit mit staatlichen Behörden auf lokaler Ebene widmen. Andererseits sollten Investitionen in die Zivilgesellschaft, mindestens in diesen Ländern, vermehrt wieder den Hilfswerken übertragen werden.

Umgekehrt müssen sich die Hilfswerke fragen, mit welchen zivilgesellschaftlichen Akteuren sie besser zusammenarbeiten können als die DEZA. Grob gesagt, liegt ihr Vorteil überall dort, wo die Arbeit politisch und konfliktträchtig ist. Die Hilfswerke müssten meines Erachtens NRO, soziale Bewegungen und Organisationen unterstützen, die in der Mobilisierung und Interessenvertretung benachteiligter Schichten stark sind, - Kräfte also, die Konflikte mit den Behörden, den herrschenden Schichten und den Gebern austragen und politische Allianzen schmieden können, um ein Gegengewicht zu bilden.

5. Die Schweiz verfolgt, wie alle Industriestaaten, eine widersprüchliche NordSüd-Politik. Die Ziele, die sie mit der Entwicklungszusammenarbeit verfolgt, stimmen nicht mit ihrer Politik im Aussenhandel oder in den globalen Finanzbeziehungen überein. Die Regierung ist sich dessen bewusst und versucht, die Kohärenz zu verbessern. Dieses lobenswerte Streben muss allerdings realistisch eingeschätzt werden. Kohärenz wird in absehbarer Zeit nicht hergestellt werden - mit andern Worten: Kohärenz wird noch auf längere Zeit lediglich den Ort bezeichnen, wo Zielkonflikte und Interessengegensätze der schweizerischen Südpolitik ausgetragen werden. Die Advocacy der Hilfswerke hakt hier ein, thematisiert die Widersprüche und versucht, schädliche politische Zielsetzungen der Regierung öffentlich zu machen und in kleinen Schritten zu korrigieren.

Viele Personen der DEZA äussern ein grosses Interesse daran, dass die Hilfswerke diese Rolle stark wahrnehmen. Die staatliche Liebe zur Advocacy privater Hilfswerke ist allerdings ambivalent. In den Verwaltungsabteilungen ausserhalb der DEZA schwindet das Verständnis dafür überaus schnell. Die politischen Kräfte im Parlament reagieren auf Advocacy-Arbeit je nach Interessenlage positiv oder ablehnend. Die Regierung hätte am liebsten eine Advocacy, die sich mit mächtigen anderen Interessen kombinieren liesse. Einige würden es ausgezeichnet finden, wenn die Hilfswerke die Marktöffnung der schweizerischen Landwirtschaft zum zentralen Thema ihrer Advocacy in der Welthandelsorganisation (WTO) machten. Eine solche Kampagne hätte den Beifall des Verbandes der schweizerischen Unternehmen (economiesuisse) und würde in 
Teilen des Bundesrates, welcher offiziell einen weitreichenden Schutz der schweizerischen Landwirtschaft verficht, auf heimliche Zustimmung stossen. Weniger beliebt ist, dass wir in Sachen WTO die internationale NRO-Kampagne aufgreifen, welche die vielfältigen, nicht mit dem totalen Freihandel vereinbaren Interessen der Entwicklungsländer thematisiert und eine grundlegende Neuorientierung des WTO-Regelwerks verlangt.

Ausserhalb der Kohärenzfragen gibt es ein Interesse der staatlichen Entwicklungsagenturen an einer allgemeineren, sozusagen volkspädagogischen Advocacy der privaten Hilfswerke. Sie sollen durch öffentliche Sensibilisierungsarbeit und Vermittlung der Problemlagen der Entwicklungsländer die Sympathien der Bevölkerung für die Entwicklungszusammenarbeit wecken und am Leben erhalten. Das ist eine Arbeit, die kommunikationstechnische Professionalität und viel Geld erfordert. Die Hilfswerke tun in dieser Richtung sehr vieles; ist es doch nicht zuletzt eine notwendige Grundlage für ihr Fundraising. Im Grundsatz besteht Einigkeit, dass die DEZA diese Sensibilisierungstätigkeit der Hilfswerke eigentlich mit unterstützen könnte und auch dürfte.

6. In der DEZA sind einige der Meinung, die Hilfswerke sollten sich aus der operativen Entwicklungszusammenarbeit zurückziehen und sich stattdessen ganz auf Advocacy konzentrieren. Ich habe oben bereits dargelegt, dass es gute Gründe für eine weitere aktive Rolle der Hilfswerke in der operativen EZA gibt. Hier will ich die Konsequenzen aufzeigen, die ein vollständiger Rückzug der Hilfswerke auf Advocacy hätte. Ich sehe deren drei: Die Hilfswerke würden an Glaubwürdigkeit verlieren. Ihre Spendeneinnahmen würden drastisch einbrechen. Die innenpolitische Basis für die EZA würde schrumpfen.

Die Advocacy-Tätigkeit stützt sich heute darauf, dass die Hilfswerke wissen, worüber sie sprechen, und vor Ort Erfahrungen machen. Wären die Hilfswerke nicht mehr in den Entwicklungsländern engagiert und würden sie ihre Beziehungen zu den Süd-Partnern verlieren, dann würden sie in den Augen der Öffentlichkeit auch einiges an Glaubwürdigkeit einbüssen.

Die Hilfswerke in der Schweiz sammeln vergleichsweise grosse Spenden auf einem hart umkämpften Spendenmarkt. Diese 250 Millionen Franken kommen zusammen, weil die Hilfswerke in den Entwicklungsländern konkrete Programme laufen haben. Die Spenderinnen und Spender wollen nicht Hilfswerke oder Advocacy finanzieren, sondern bedürftigen Menschen im Süden helfen. Beim Fundraising stehen deshalb die EZA-Aktivitäten im Vordergrund. Würden diese wegfallen, so würden die Spenden auf einen Bruchteil zusammenschrumpfen. Wir können dies mit den Spendeneinnahmen illustrieren, welche die Erklärung von Bern als eine reine Advovcacy-Organisation erzielt. Sie bringt es auf etwa 2,5 Millionen Franken, und sogar sie benutzt für ihre Spendenkampagne lieber konkrete Themen wie ihr Engagement im fairen Handel (Clean Clothes Campaign), als ihr abstrakteres und einem Massenpublikum schwieriger vermittelbares Engagement in WTO-Angelegenheiten. Würden alle Hilfswerke zu „Erklärungen von Bern“ mutieren, brächten sie es im besten Fall auf zehn, fünfzehn Millionen Franken, und die meisten Hilfswerke würden wohl untergehen.

Mit reduzierter Glaubwürdigkeit, einer kleineren Zahl von Hilfswerken und viel weniger Geld würde auch die innenpolitische Basis für die Entwicklungs- 
zusammenarbeit langsam erodieren. Kurz zusammengefasst: Der Rückzug der Hilfswerke aus der operativen EZA auf reine Advocacy wäre ein Meisterstreich an politischer und betriebswirtschaftlicher Dummheit. 